\title{
Anti-EGFRvIII/CD3 BiTE Antibody AMG 596
}

National Cancer Institute

\section{Source}

National Cancer Institute. Anti-EGFRvIII/CD3 BiTE Antibody AMG 596. NCI Thesaurus.

Code C146824.

A proprietary recombinant bispecific T-cell eng ager (BiTE) antibody composed of two single-chain variable fragments (scFv), one that is directed against a tumor-associated antigen (TAA), the epidermal growth factor receptor (EGFR) deletion-mutant form, EGFR variant III (EGFRVIII), and one that is directed against the CD3 antigen found on Tlymphocytes, with potential immunostimulating and antineoplastic activities. Upon administration of anti-EGFRVIII/CD3 BiTE antibody AMG 596, the bispecific antibody binds to both the CD3 antigen on cytotoxic T-lymphocytes (CT Ls) and EGFRvIII found on EGFRvIII-expressing tumor cells. This activates and crosslinks CT Ls with EGFRvIIIexpressing tumor cells, which results in the CT L-mediated cell death of EGFRvIIIexpressing tumor cells. EGFRvIII, a mutation in the EGFR gene where exons 2-7 have been deleted, is overexpressed by a variety of cancers, but is absent in normal, healthy cells. It plays a key role in tumor cell proliferation, tumor angiogenesis and resistance to both radio- and chemotherapy. 